\title{
Performance Analysis of Dynamic Spectrum Access using Composite Fading Channel
}

\author{
Partha Pratim Paul \\ Lecturer, Department of Electrical and Computer Engineering, Presidency University, Dhaka, Bangladesh
}

\begin{abstract}
In this paper, a composite multipath or shadowed fading environment consists of multipath fading superimposed on shadowing is considered. In this environment the receiver does not average out the envelope fading due to multipath but rather reacts to the instantaneous composite multipath/shadowed signal [6]. And the most important point is it is difficult to perform power control required for DSA based on fast fading, so it is proposed to base its solely on shadow fading with some power back off. Otherwise, the PR has to transmit pilots very often, which is not rational. There are many approaches and various combinations suggested in the literature for obtaining the composite distribution but few approaches that have been proposed to attain the objective using composite distributions.
\end{abstract}

Keywords: Antenna Selection, Fading Channel, Outage Probability, Cooperative Relay Networks, Amplify - and Forward Relaying, Decode - and - Forward (DF), Multiple - Input Multiple - Output

\section{INTRODUCTION}

With the increasing demand for Wireless Communication Systems and Services, radio spectrum has become a scarce resource, which has grown tremendously over last few decades. So the need for more efficient utilization of this precious resource has led to various spectrum sharing protocols [1-5]. Hence, building cognitive radio systems support dynamic access to the available spectrum has appeared recently as a novel solution for the huge expansion of wireless system. It is attaining communication paradigm to effectively address spectrum scarcity challenge. The basic concept here is to employ a hierarchical model so that both the systems termed as primary users (Licensed) and secondary users (Unlicensed) co-exist together in the same frequency band under the constraint that the presence of secondary users does not limit primary system performance. Therefore the objective of secondary users is to exploit the inactive sessions of the primary users termed as spectral holes in opportunistic fashion. Alternatively, cognitive users can exploit spatial holes in the spectrum. In dynamic spectrum sharing type of cognitive radio protocol for example, the cognitive radios are allowed to use the spectrum as long as its transmission does not result in degradation of the performance of primary system beyond a prescribed limit $[2,5]$.

In this paper, a composite multipath or shadowed fading environment consists of multipath fading superimposed on shadowing is considered. In this environment the receiver does not average out the envelope fading due to multipath but rather reacts to the instantaneous composite multipath/shadowed signal [6]. And the most important point is it is difficult to perform power control required for DSA based on fast fading, so it is proposed to base its solely on shadow fading with some power back off. Otherwise, the PR has to transmit pilots very often, which is not rational. There are many approaches and various combinations suggested in the literature for obtaining the composite distribution but few approaches that have been proposed to attain the objective using composite distributions.

\section{SYSTEM MODEL}

In this scheme the system model consists of two terminals (PT and PR) in the primary system and two terminals (ST and SR) in the secondary system which are all having a single antenna. Here secondary transmit antenna tries to interfere the signal with primary channel. Here, primary power source PT transmits with power, $\mathrm{P}_{\mathrm{T}}$ and secondary power source ST transmits with power, $\mathrm{P}_{\mathrm{S}}$.

Here we are considering primary power source broadcasts its information only to the primary receiver while secondary power source broadcasts its information both to secondary receiver and primary receiver. The signal transmits from the primary transmitter and secondary transmitter $\mathrm{y}_{\mathrm{a} 1}$ and $\mathrm{y}_{\mathrm{a} 2}$ respectively to the destination primary receiver, can be written as

$$
\mathrm{y}_{\mathrm{a} 1}=\sqrt{\mathrm{P}_{\mathrm{P}}} \mathrm{h}_{\mathrm{pp}} \mathrm{x}_{\mathrm{PT}}+\mathrm{n}_{\mathrm{p}}
$$




$$
\mathrm{y}_{\mathrm{a} 2}=\sqrt{\mathrm{P}_{\mathrm{S}}} \mathrm{gx}_{\mathrm{ST}}+\mathrm{n}_{\mathrm{s}}
$$

in which $\mathrm{x}_{\mathrm{PT}}$ and $\mathrm{x}_{\mathrm{ST}}$ is the transmitted information symbol from primary and secondary transmitter respectively and $\mathrm{n}_{\mathrm{p}}$ and $\mathrm{n}_{\mathrm{s}}$ are the additive noises.

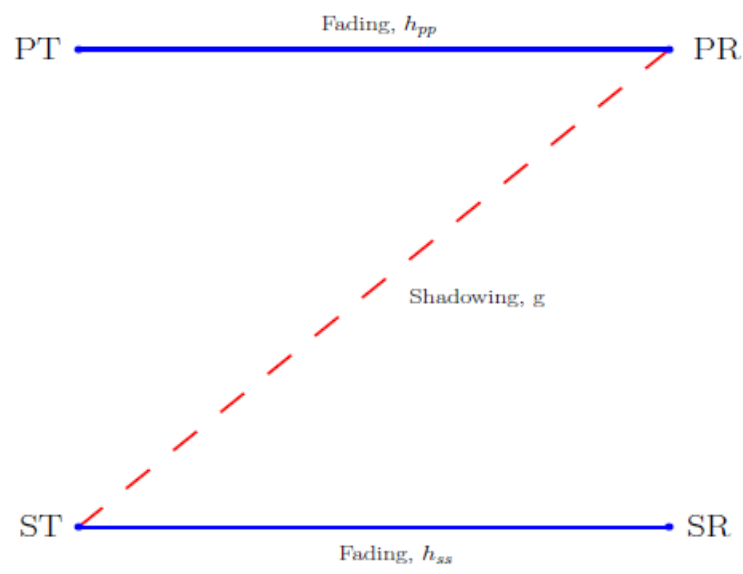

Fig. 1 Dynamic Spectrum Access System Model

From the above two equations, $\mathrm{h}_{\mathrm{pp}}$ is the fading channel co-efficient from primary transmitter to primary receiver and $\mathrm{g}$ is the shadowing channel co-efficient from secondary transmitter to primary receiver. They are modeled as zeromean, complex Gaussian random variables with variances $\sigma_{\text {PT }}^{2}$ and $\sigma_{\text {ST }}^{2}$ respectively. The noise terms $n_{p}$ and $n_{s}$ are modeled as zero-mean complex Gaussian random variables with variance $N_{0}$. So with the knowledge of $h_{p p}$ and $g$ channel co-efficient, the total received signal at primary receiver is

$$
\mathrm{y}_{\mathrm{PR}}=\sqrt{\mathrm{P}_{\mathrm{P}}} \mathrm{h}_{\mathrm{pp}} \mathrm{x}_{\mathrm{PT}}+\sqrt{\mathrm{P}_{\mathrm{S}}} \mathrm{gx}_{\mathrm{ST}}+\mathrm{n}
$$

Now we need to calculate the total instantaneous signal-to-noise ratio at the destination. It can be written as

$$
\mathrm{SNR}=\Gamma\left|\mathrm{h}^{2}\right|
$$

where $I=\frac{P}{N_{0}}$ in which $P$ is the power source from respective node and $N_{0}$ is the noise. The SNR receives at the destination is the sum of the SNRs from the primary source and secondary source. So we can write

$$
\digamma_{\mathrm{PR}}=\frac{\mathrm{P}_{\mathrm{P}}\left|\mathrm{h}_{\mathrm{pp}}\right|^{2}}{\mathrm{P}_{\mathrm{S}}|\mathrm{g}|^{2}+\sigma^{2}}
$$

Similarly, received signal at secondary receiver side can be written as

So, we can write

$$
\mathrm{y}_{\mathrm{SR}}=\sqrt{\mathrm{P}_{\mathrm{S}}} \mathrm{h}_{\mathrm{SS}} \mathrm{x}_{\mathrm{ST}}+\mathrm{n}_{\mathrm{s}}
$$

$$
\Gamma_{S R}=\frac{P_{S}\left|h_{S S}\right|^{2}}{\sigma^{2}}
$$

\section{OUTAGE PROBABILITY FOR COMPOSITE FADING CHANNEL}

A. When Primary channel is Exponential Distribution and Interfering channel is Lognormal Distribution

From the system model, shown in the fig (1), we have considered exponential distribution for primary channel and lognormal distribution for secondary to primary channel. Using the equation (5), The PDFs of the desired and interfering signal can be written as

$$
\begin{aligned}
\mathrm{f}_{\left|h_{\mathrm{PP}}\right|^{2}}(\mathrm{x}) & =\lambda \mathrm{e}^{-\mathrm{x} \lambda} \\
\mathrm{f}_{|\mathrm{g}|^{2}}(\mathrm{x}) & =\frac{1}{\sigma \gamma \sqrt{2 \pi}} \exp \left[-\frac{\left(10 \log _{10} \gamma-\mu\right)^{2}}{2 \sigma^{2}}\right]
\end{aligned}
$$


Vol. 8, Issue 4, April 2019

The bottleneck of lognormal distribution is to obtain the closed form expression, hence making the performance evaluation of communication links over such channels very challenging. In order to obtain a more tractable expression, the lognormal shadowing is approximated by the Inverse-Gaussian (IG) distribution which is given by [33]

$$
f_{Y}(y)=\sqrt{\frac{\lambda}{2 \pi}} y^{-\frac{3}{2}} \exp \left[-\frac{\lambda(y-\theta)^{2}}{2 \theta^{2}} y\right], \quad y>0
$$

The parameters $\lambda$ and $\theta$ are linked to $\mu$ and $\sigma$ by matching the first and second moments of the lognormal distribution with the first and the second moments of the IG distribution as follows

leading to

$$
f(x)=\left\{\begin{array}{c}
\exp \left(\mu+\frac{\sigma^{2}}{2}\right)=\theta \\
\exp \left(2\left(\lambda+\sigma^{2}\right)\right)=\theta
\end{array}\right.
$$

So, the new approximated PDF of the interferring signal is

$$
f(x)=\left\{\begin{array}{l}
\lambda=\frac{\exp (\mu)}{2 \sinh \left(\frac{\sigma^{2}}{2}\right)} \\
\theta=\exp \left(\mu+\frac{\sigma^{2}}{2}\right)=\theta
\end{array}\right.
$$

$$
f_{|g|^{2}}(x)=\sqrt{\frac{\lambda}{2 \pi}} y^{-\frac{3}{2}} \exp \left[-\frac{\lambda(y-\theta)^{2}}{2 \theta^{2}} y\right], \quad y>0
$$

As the CDF of Exponential Distribution is,

$$
\mathrm{f}_{\left|\mathrm{h}_{\mathrm{PP}}\right|^{2}}(\mathrm{x})=1-\mathrm{e}^{-\mathrm{x} \lambda}
$$

using expression (11) and (12), the outage probability can be found as,

$$
\begin{aligned}
\mathrm{P}_{\mathrm{out}} & \left.=\operatorname{Pr} \frac{\mathrm{P}_{\mathrm{P}}\left|\mathrm{h}_{\mathrm{pp}}\right|^{2}}{\mathrm{P}_{\mathrm{S}}|\mathrm{g}|^{2}+\sigma^{2}}<\gamma_{\mathrm{th}}\right] \\
& \left.=\operatorname{Pr}\left|\mathrm{h}_{\mathrm{pp}}\right|^{2}<\frac{\gamma_{\mathrm{th}} \mathrm{P}_{\mathrm{S}}\left(|\mathrm{g}|^{2}+\sigma^{2}\right)}{\mathrm{P}_{\mathrm{S}}}\right] \\
& =\int_{0}^{\infty} \int_{0}^{\mathrm{I}_{\mathrm{PR}}}\left[\mathrm{F}_{\left|\mathrm{h}_{\mathrm{PP}}\right|^{2}}(\mathrm{x}) \mathrm{dx \textrm {x } _ { | \mathrm { g } | ^ { 2 } } ( \mathrm { y } ) \mathrm { dy } ]}\right.
\end{aligned}
$$

Using Appendix A, the outage probability can be written as,

$$
\mathrm{P}_{\text {out }}=1-\sqrt{\frac{\lambda_{\mathrm{g}}}{2 \pi}}\left(\exp \left[-\frac{\gamma_{\text {th }}}{\mathrm{P}_{\mathrm{p}} \Omega_{\mathrm{h}}}\right]\right)
$$

\section{SIMULATION RESULT}

In this section performance at the primary receiver and second receiver both will be checked with respect to both desired signal and interfering transmitter in terms of the outage probability. Also performance at the primary receiver is checked with respect to secondary transmitter in terms of the outage probability.

A. Outage Probability at Primary Receiver when no interference

Here the outage probability of the primary system at the primary receiver is simulated with respect to desired signal considering the distance between the primary terminals (PT and PR) $d_{P}$ equal to 1 , where all these distances are the normalized distances with respect to the distance between the primary terminals (PT, PR). Fig. 2 shown plots of the outage probability against $\mathrm{SNR}$ of the primary system $\mathrm{P}_{\mathrm{t}} / \mathrm{N}_{0}$ equals to $35 \mathrm{~dB}$.

In fig. 2, we can observe that the outage probability of the primary system plotted against the primary power when there is no interference signal. And as expected, with the increase of power from the primary side, the outage probability is also decreased. 


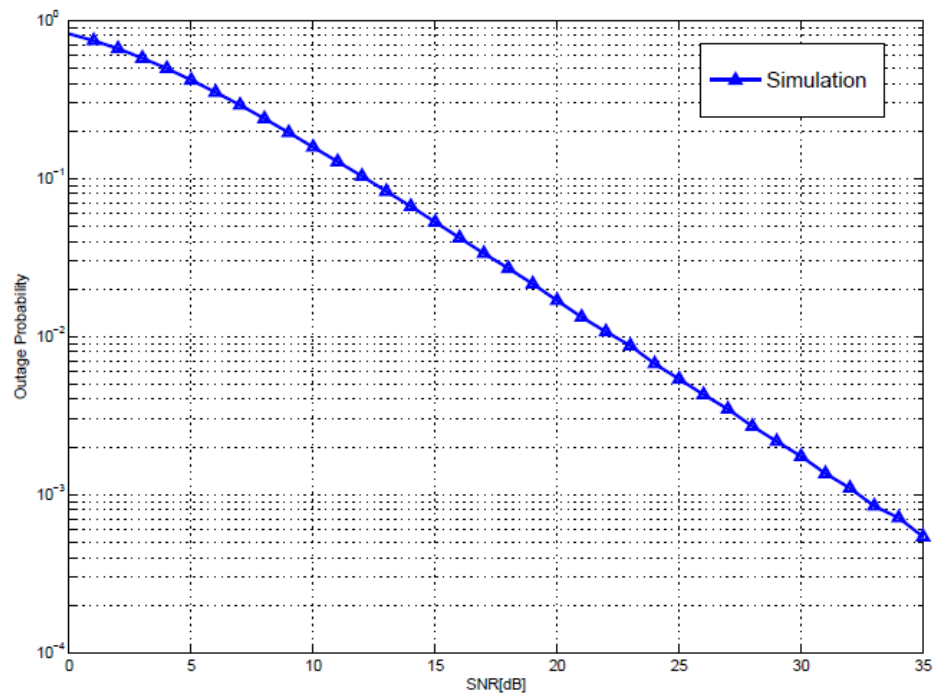

Fig. 2 Outage probability at Primary Receiver v/s SNR at Primary Transmitter

B. Outage Probability at Primary Receiver for single interferer

Here the outage probability of the primary system at the primary receiver is simulated when interfering transmitter has started transmitting power.

Here distance between the primary terminals (PT and PR) $d_{P}$ equal to 1 and distance between the secondary transmitter to primary terminals (ST and PR) $d_{P S}$ equal to 5, where all these distances are the normalized distances with respect to the distance between the primary terminals (PT, PR). Fig. 3 shown plots of the outage probability against SNR of the primary system $P_{t} / N_{0}$ equals to $70 \mathrm{~dB}$.

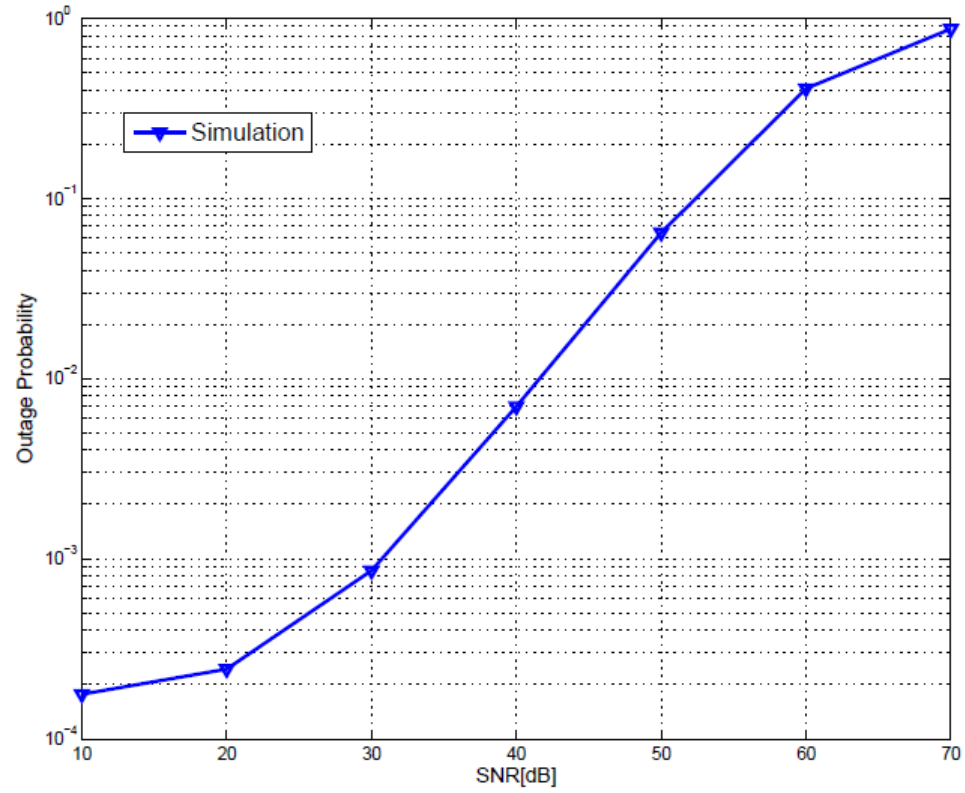

Fig.3 Outage probability at Primary Receiver v/s SNR at Secondary Transmitter for single interferer

In fig. 3, we can observe that the outage probability of the primary system plotted against the increase of secondary power and there is single interference signal. And as expected, with the increase of power from the secondary transmitter, the outage probability is increased at primary receiver.

C. Outage Probability at Secondary Receiver for fixed primary outage

Here the outage probability of the secondary system at the secondary receiver is simulated when the primary outage is fixed from the primary receiver side. Distance considered between the secondary terminals (ST and SR) $d_{S}$ equal to 1 , where all these distances are the normalized distances with respect to the distance between the primary terminals (ST, SR). Fig. 4 shown plots of the outage probability against SNR of the secondary system $S_{t} / N_{0}$ equals to $30 \mathrm{~dB}$. 
Vol. 8, Issue 4, April 2019

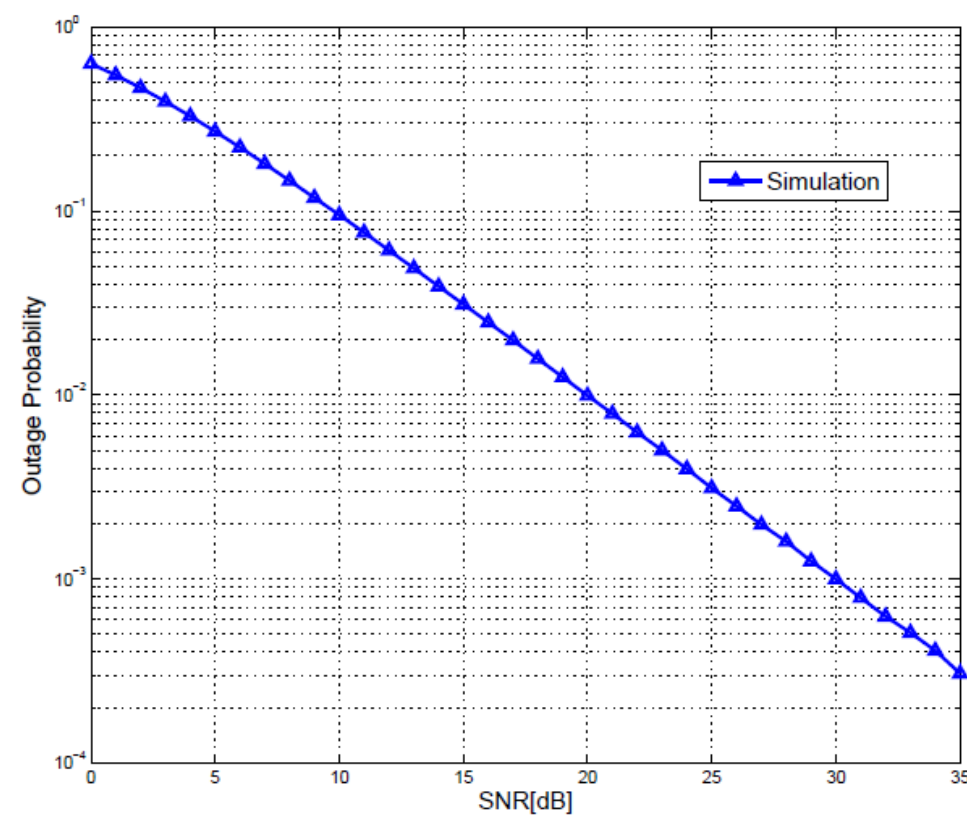

Fig.4 Outage probability at Secondary Receiver v/s SNR at Secondary Transmitter for fixed primary outage

In fig. 4, it is also observed that the outage probability of the secondary system plotted against the increase of secondary power. With the increase of power from the secondary transmitter, the outage probability is decreasing at secondary receiver.

D. When Primary channel is Exponential Distribution and Interfering channel is Lognormal Distribution

Here the outage probability of the primary system is simulated considering the distance between the primary terminals (PT and PR) $d_{P}$ equal to 1, distance between secondary terminal (ST) to PR as da equal to 5, where all these distances are the normalized distances with respect to the distance between the primary terminals (PT, PR). The fig. 5 has shown plots the outage probability against $\mathrm{db}$ for the SNR of the primary system $P_{t} / N_{0}$ equals to $30 \mathrm{~dB}$.

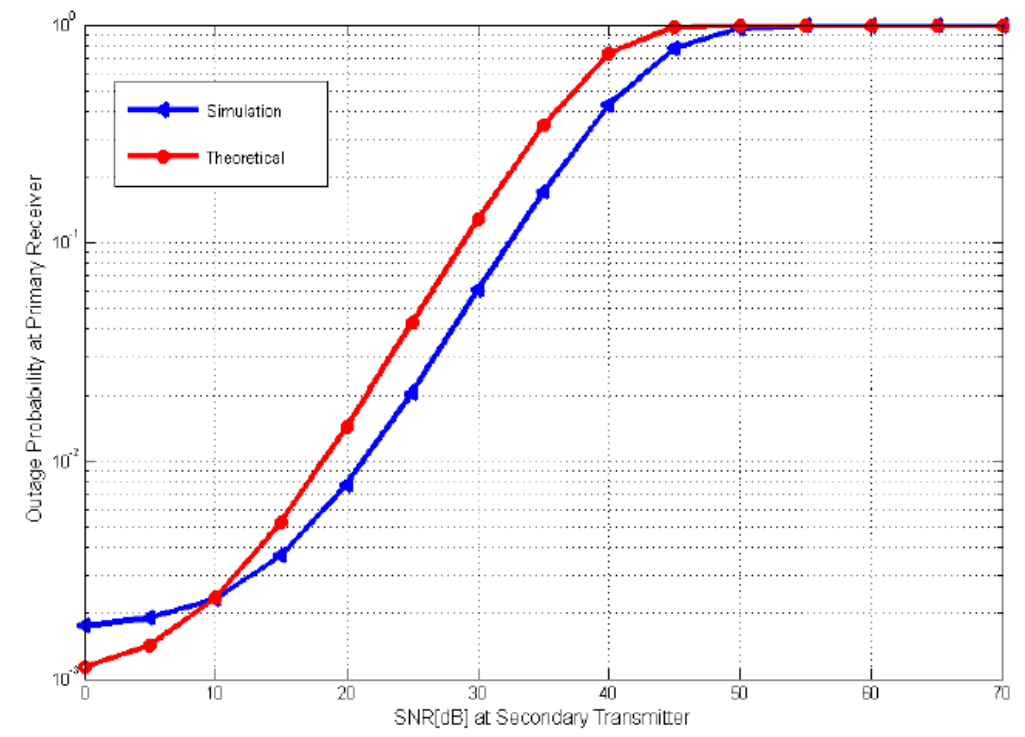

Fig. 5 Outage probability at Primary Receiver v/s SNR at Secondary Transmitter

In this figure we can observe that the outage probability of the primary system plotted both analytically and with the CDF expression appear to be nearly matching which proves the correctness of the CDF expression derived. The deviation, which is appeared, this is due to the taken approximation of lognormal distribution to Inverse-Gaussian distribution. Also we can observe that the outage probability is increasing at the primary receiver when SNR of secondary transmitter is increasing, so the interference at the primary side is becoming high. 


\section{APPENDIX A}

$$
\int_{0}^{\infty} \mathrm{x}^{\mathrm{v}-1} \mathrm{e}^{-\frac{\beta}{\mathrm{x}}-\gamma \mathrm{x}} \mathrm{dx}=2\left(\frac{\beta}{\gamma}\right)^{\frac{\mathrm{v}}{2}} \mathrm{k}_{\mathrm{v}}(2 \sqrt{\beta \gamma})
$$

\section{CONCLUSION}

We have analyzed to get the outage performance with composite distribution. The general case was shown to be intractable. However, when the primary channel is exponential, performance of the secondary can be analyzed when secondary channels are of composite type. Specifically, we use the inverse Gaussian approximation to analyze the performance of the DSA system with exponential channel in the primary and composite channel in the secondary.

\section{REFERENCES}

[1]. J. Mitola, “Cognitive Radio : An integrated agent architecture for software defined radio, Ph.D. dissertation, KTH, Stockholm, Sweden, Dec., 2000 .

[2]. Y. Xing, C. N. Mathur, M. A. Haleem, R. Chandramouli, and K. P. Subbalakshmi, "Dynamic spectrum access with QoS and interference temperature constraints, IEEE Trans. Mobile Comput., vol. 6, no. 4, pp. 423-433, Apr. 2007.

[3]. I. Mari, A. Goldsmith, G. Kramer, and S. Shamai, "On the capacity of interference channels with a cognitive transmitter, in Proc. Inf. Theory Appl. Workshop, Jan. 2007, pp. 268-273.

[4]. A. Joviei and P. Viswanath, "Cognitive radio: An information-theoretic perspective, IEEE Trans. Inf. Theory, vol. 55, no. 9, pp. 3945-3958, Sep. 2009.

[5]. Goldsmith, A.; Jafar, S.A.; Maric, I.; Srinivasa, S., "Breaking Spectrum Gridlock With Cognitive Radios: An Information Theoretic Perspective," Proceedings of the IEEE, vol.97, no.5, pp.894-914, May 2009.

[6]. G. L. Stuber, Principles of Mobile Communications. Norwell, MA: Kluwer Academic Publishers, 1996.

\section{BIOGRAPHY}

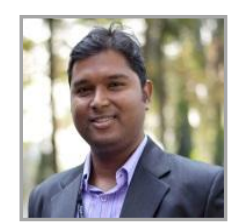

Partha Pratim Paul received the M. Tech degree in Communications Engineering from the Indian Institute of Technology Delhi, New Delhi, India, in 2014. He joined the Presidency University, Dhaka, Bangladesh, in 2016, where he is currently working as Lecturer in Department of Electrical and Computer Engineering. His interests are in cognitive radio, cooperative communication, MIMO systems, physical layer security, wireless energy harvesting etc. 